(C) 1996 IEEE. Personal use of this material is permitted. However, permission to reprint/republish this material

for advertising or promotional purposes or for creating new collective works for resale or redistribution to servers

or lists, or to reuse any copyrighted component of this work in other works must be obtained from the IEEE.

\title{
RF SYSTEM MODELING \\ FOR THE HIGH AVERAGE POWER FEL AT CEBAF *
}

\author{
L. Merminga, J. Fugitt, G. Neil and S. Simrock, CEBAF, 12000 Jefferson Ave, Newport News, VA 23606 USA
}

Abstract

High beam loading and energy recovery compounded by the use of superconducting cavities, which requires tight control of microphonic noise, place stringent constraints on the linac rf system design of the proposed high average power FEL at CEBAF. Longitudinal dynamics imposes off-crest operation, which in turn implies a large tuning angle to minimize power requirements. Amplitude and phase stability requirements are consistent with demonstrated performance at CEBAF. A numerical model of the CEBAF rf control system is presented and the response of the system is examined under large parameter variations, microphonic noise and beam current fluctuations. Studies of the transient behavior lead to a plausible start-up and recovery scenario.

\section{RF SYSTEM OVERVIEW}

The driver accelerator for the high average power FEL, proposed for construction at CEBAF, is a recirculating energyrecovering $200 \mathrm{MeV}, 5 \mathrm{~mA} \mathrm{cw}$ superconducting $\mathrm{rf}$ (SRF) electron accelerator. The accelerator consists of a $10 \mathrm{MeV}$ injector, a 96 MV SRF linac with a two-pass recirculation transport which accelerates the beam to $200 \mathrm{MeV}$, decelerates it for energy recovery through two passes, and transports it to a $\sim 10 \mathrm{MeV}$ dump [1].

Matching of the longitudinal phase space for bunching going into the wiggler and debunching going out of the wiggler and into energy recovery, implies a fairly restrictive set of constraints on the rf voltage, phases of the four beams (two accelerating and two decelerating) with respect to the crest of the rf wave, and arc compaction factors $\left(M_{56}\right)$. Phasing of the four beams is such that the resultant beam vector has a strong reactive component; therefore the rf cavities must be operated off resonance to minimize the required generator power. With energy recovery, the generator power that must be supplied to the cavities is greatly reduced to approximately $1.5 \mathrm{~kW}$ per cavity, despite accelerating $5 \mathrm{~mA}$ by $4 \mathrm{MV}$.

The rf system provides power for acceleration of the electron beam, and control of the phase and amplitude of the accelerating field. High beam loading, energy recovery and the use of superconducting cavities, which require tight control of microphonic noise, place stringent constraints on the linac rf system design. A dedicated klystron, power amplifier and regulation system for each rf accelerating cavity is required because of the large influence of microphonic noise parametrically modulating the resonant frequency of the superconducting cavity. This modulation is not coherent over the many cavities, and results in random errors in phase and amplitude that can best be corrected by the use of individual rf cavity control systems.

To minimize cost and risk it has been proposed that the CEBAF rf control system [2] be used for the FEL driver accelerator. To

\footnotetext{
* Supported by the Virginia Center for Innovative Technology and DOE Contract \# DE-AC05-84ER40150.
}

Table I

RF system requirements

\begin{tabular}{|l|l|}
\hline Parameter & Requirement \\
\hline RF power to beam per cavity & $1.34 \mathrm{~kW}$ \\
Klystron power per cavity & $5 \mathrm{~kW}$ \\
Phase stability (rms) & $0.14^{0}$ \\
Phase stability (long term) & $3^{0}$ \\
Gradient stability (rms) & $2.8 \times 10^{-4}$ \\
Gradient stability (long term) & $1.4 \times 10^{-3}$ \\
Gradient & $8 \mathrm{MV} / \mathrm{m}$ \\
Accelerating phases & $1.8^{0},-13.5^{0}, 195.3^{0}, 180^{0}$ \\
Loaded Q & $6.6 \times 10^{6}$ \\
Tuning angle & $-61.5^{0}$ \\
\hline
\end{tabular}

test the control system capabilities and its robustness under the FEL operating conditions, we developed a model of the control system using SIMULINK [3], which numerically integrates the equations of motion of the system. This paper describes the model and presents results of the simulations.

We start with a summary of the FEL rf system requirements for the linac. The cavity equation is presented next and power requirements for the linac at steady-state are derived. RF amplitude and phase control is addressed next. We describe the rf model, discuss its validity and present simulation results which include transient behavior, regulation of microphonics, response to large parameter variation and a start-up/recovery scenario.

\section{RF SYSTEM REQUIREMENTS}

Table I summarizes the rf system requirements for the linac.

\section{STEADY-STATE: POWER REQUIREMENTS}

The rf cavity powered by an rf source (klystron) can be represented by a resonant LCR circuit [4]. The beam in the cavity is represented by a current generator. The dynamics of this system can be described, to a very good approximation, by the following first order differential equation,

$$
\frac{d \tilde{v}_{c}}{d t}+\frac{\omega_{0}}{2 Q_{L}}(1-i \tan \Psi) \tilde{v}_{c}=\frac{\omega_{0} R_{L}}{4 Q_{L}}\left(\tilde{i}_{g}-\tilde{i}_{b}\right)
$$

where $\omega_{0}$ is the cavity resonant frequency, $Q_{L}$ is the loaded $Q$ of the cavity and $R_{L}$ is the loaded shunt impedance $R_{L}=(r / Q) Q_{L}$. For CEBAF cavities $(r / Q)=480 \Omega$ per cavity. In arriving at (1) we assume that the cavity voltage, generator and beam current vary as $e^{i \omega t}$, where $\omega$ is the rf frequency, and $\tilde{v}_{c}, \tilde{i}_{g}$ and $\tilde{i}_{b}$ are the corresponding complex amplitudes, varying slowly with time. For short bunches, $i_{b} \approx 2 I_{0}$, where $I_{0}$ is the average beam current, and $i_{b}$ denotes the magnitude of $\tilde{i}_{b}$. In this equation $\Psi$ is the tuning angle defined by $\tan \Psi=-2 Q_{L}\left(\omega-\omega_{0}\right) / \omega_{0}$. 
The current source $\tilde{i_{b}}$ is the vector sum of the four beams present in the linac cavities each with an average current of $I_{0}=$ $5 \mathrm{~mA}$ and phases with respect to the crest of the rf wave, $\phi_{k}, k=$ $1,2,3,4$. Therefore $\tilde{i}_{b}=2 I_{0} \sum_{k=1}^{4} e^{i \phi_{k}}$ or $\tilde{i}_{b}=2 I_{b} e^{i \Psi_{b}}$ where $2 I_{b}$ is the magnitude and $\Psi_{b}$ the phase of $\tilde{i}_{b}$. Similarly we write $\tilde{v}_{c}=v_{c} e^{i \Psi_{c}}$. For convenience the reference phase is taken in the direction of $\tilde{v}_{c}$, therefore $\Psi_{c}=0$. In steady-state the generator power is given by

$$
P_{g}=\frac{(1+\beta)}{16 \beta} i_{g}^{2} R_{L}
$$

where $\beta$ is the cavity coupling coefficient. Using eq. (1) we can express the generator power in terms of $\Psi, I_{b}, \Psi_{b}$ and $\beta$, and obtain the condition for optimum tuning, $\tan \Psi_{\text {opt }}=$ $\left(I_{b} R_{L} / v_{c}\right) \sin \Psi_{b}$. The generator power at optimum tuning is

$$
P_{g 0}=\frac{v_{c}^{2}}{R_{L}} \frac{(1+\beta)}{4 \beta}\left[1+\frac{I_{b} R_{a}}{V_{c}} \cos \Psi_{b}\right]^{2} .
$$

For the accelerating phases given in Table I, $I_{b}=2.33 \mathrm{~mA}$ and $\Psi_{b}=-89^{0}$. For $Q_{L}=6.6 \times 10^{6}, Q_{0}=5 \times 10^{9}$, and $v_{c}=4 \mathrm{MV}$, the optimum tuning angle is $-61.5^{0}$, and the required generator power is equal to $1.34 \mathrm{~kW}$ per cavity.

\section{RF AMPLITUDE AND PHASE CONTROL}

Several designs exist to control the rf fields in superconducting cavities. The "classical" approach, employed by CEBAF, uses separate control of amplitude and phase. In the CEBAF system the cavity signal at $1497 \mathrm{MHz}$ is downconverted to an IF frequency of $70 \mathrm{MHz}$ where the phase detector and the controllers for amplitude and phase are operated. The amplitude of the accelerating field is determined by a Schottky detector which is operated in its linear range; i.e., the output voltage is proportional to the accelerating field. The fast phase detector uses an ana$\log$ multiplier. The output signal is proportional to the $\sin (\Delta \phi)$, where $\Delta \phi$ is the phase difference between the rf reference (at 70 $\mathrm{MHz}$ ) and the frequency-converted field probe (rf signal). Amplitude and phase modulators use analog multipliers at $70 \mathrm{MHz}$.

The gains and the frequency response of the feedback loops have to be optimized to minimize the residual amplitude and phase noise under steady-state conditions. During tune-up of the accelerator the field stability requirements can be relaxed but the control system must be stable for a wide range of beam loading conditions. The stability over a wide range of parameters determines the robustness of the rf control system. The coupling between the amplitude and phase loop should be minimal for maximum stability (robustness) and minimum residual noise.

Microphonic noise modulates the resonance frequency, which results in the uncontrolled (no feedback) case in rms phase variations of up to $7^{0}$ and amplitude fluctuations of $0.5 \% \mathrm{rms}$ (average tuning angle zero) or $8.7 \% \mathrm{rms}$ (average detuning angle $45^{\circ}$ ). For the required stability a minimum gain of $40 \mathrm{~dB}$ for the phase loop and $50 \mathrm{~dB}$ for the amplitude loop is required. The typical microphonic noise frequency range is from $1 \mathrm{~Hz}$ to 200 $\mathrm{Hz}$ for the CEBAF accelerator.

\section{A. RF Modeling}

To simulate the performance of the CEBAF rf control system with FEL operating conditions, we developed a model of the cavity and low level controls using SIMULINK, a MATLAB program for simulating dynamic systems. Figure 1 is a graphical representation of the rf model of the CEBAF rf control system. Next we give a detailed description of the model.

Equation (1) describes the interaction of the cavity fields with the beam and generator currents. The model includes microphonic noise in the form of sinusoidal modulation of the cavity's resonant frequency, $\delta \omega=\omega_{M} \sin \left(\omega_{f} t\right)$ where $\omega_{M}$ is the amplitude and $\omega_{f}$ the frequency of the noise. In addition we include the Lorentz-force detuning as another first order equation [5]

$$
\tau_{m} \dot{\Delta \omega}=-\Delta \omega-2 \pi k v_{c}^{2}
$$

where $k=3 \mathrm{~Hz} /(\mathrm{MV} / \mathrm{m})^{2}, v_{c}$ is the cavity gradient in $\mathrm{MV} / \mathrm{m}$, and $\tau_{m}$ is the mechanical time constant of the cavity, equal to $0.5 \mathrm{msec}$. The current source is the sum of the generator and beam current. The klystron power is limited to $4 \mathrm{~kW}$ (linear range). Outputs of the cavity model are the amplitude and phase of the cavity voltage. The amplitude signal is compared to the amplitude setpoint and the normalized error signal is amplified by the loop gain. The loop gain is given by $C(s)=H(s)[1+G(s)]$ where $H(s), G(s)$ are the transfer functions of the broadband and lowfrequency gain respectively, $H(s)=\frac{K_{1}}{1+s T_{1}}, \quad G(s)=\frac{K_{2}}{1+s T_{2}}$, $K_{1}=100$ and $\left(2 \pi T_{1}\right)^{-1}=1 \mathrm{MHz}$, and $K_{2}=30$ and $\left(2 \pi T_{2}\right)^{-1}=$ $200 \mathrm{~Hz}$. The broadband gain of 100 (up to $1 \mathrm{MHz}$ ) is boosted by an additional low-frequency gain of 30 which allows for an error reduction by a factor of 3000 for frequencies up to $200 \mathrm{~Hz}$. However during start-up, the low-frequency gain is turned off since it saturates the modulator drive (to be described later). This combination of broadband and low-frequency gains provides fast settling times in pulsed mode, suppression of microphonics to very low levels, and maintains an energy-gain stability of better than $10^{-3}$ when the average beam current is increased from low currents to full beam loading. The Bode plot of the system, shown in fig. 2, includes three additional poles at $3 \mathrm{MHz}$, contributed from the klystron hardware, as well as the cavity pole which occurs at $125 \mathrm{~Hz}$ (on resonance). It shows that unity gain is reached at $100 \mathrm{kHz}$ with a phase margin of $50^{\circ}$. 
The controller for the phase of the accelerating field employs a vector modulator. The two inputs control the in-phase (real) and quadrature (imaginary) (I/Q control) components of the incident wave. The in-phase input is set to a fixed bias voltage of $5 \mathrm{~V}$, while the quadrature input is used to control the cavity phase error. A control voltage range of $\pm 5 \mathrm{~V}$ allows therefore for a phase control range of $\pm 45^{0}$ which is the sufficient for the microphonics observed at CEBAF. The vector modulator has the inverse transfer function of the cavity. The amplitude is increased as function of phase as $A / A_{0}=\sqrt{1+\tan ^{2} \Psi}$ therefore exactly compensating the reduced gradient when the cavity is detuned by an angle $\Psi$. Phase control by itself stabilizes the amplitude if the origin of the phase noise is purely microphonics, and if the cavity is operated on resonance. The combination of amplitude control and phase control using the quadrature component of the incident wave is identical to I/Q control if the quadrature component of the cavity voltage is zero. In this case the amplitude controller controls only the in-phase component of the incident wave.

\section{B. Validity of Model}

To test the validity of the model, it was used to predict the magnitude of induced transients when $200 \mu \mathrm{A}$ of beam is suddenly turned off. These transient beam loading measurements have been performed at CEBAF and data are available for comparison [6]. The experimentally observed transients were at the $1 \times 10^{-3}$ level, while the rf model predicted $1.3 \times 10^{-3}$. In addition the shape of the beam generated voltage fluctuation is similar and the recovery time is in very good agreement with the data. More extensive studies of the validity of the model are planned.

\section{Simulation Results}

Extensive simulation studies were carried out to examine the behavior (stability, robustness) of the rf system: Studies of the transient behavior led to a plausible start-up and recovery scenario which is described next. Once steady-state has been established, the system response to large parameter variations was examined. At steady-state, control of microphonic noise and beam current fluctuations were evaluated.

a) Start-up and recovery

The gradients in the cavities are ramped to nominal $8 \mathrm{MV} / \mathrm{m}$. The magnetostrictive tuners maintain on-resonance condition by regulating the phase between incident and transmitted signal to zero. This phase coincides with the tuning angle $\Psi$ for zero beam current. As the beam current is raised slowly, the true tuning angle will change according to eq. (1) of [7], which correspnds to the condition of optimum tuning and is maintained for all beam loading scenarios. It is therefore not necessary to calculate and control the true tuning angle.

\section{b) Response to large parameter variations}

We examined the response of the system to the following large, step-function-like perturbations: 1) Change of the tuning angle from $-61.5^{0}$ to $-41.5^{0}$. 2) Change of the phase of both decelerating beams by $\pm 2^{0}$. 3) Reduction of the beam current of the two decelerating beams by $1 \%$. 4) Reduction of the gradient setpoint by $1 \%$. In all cases we calculated the magnitude of induced transients on the phase and amplitude of the accelerating field. In most cases the induced voltage fluctuations are within the requirements outlined earlier. The system appears to quickly

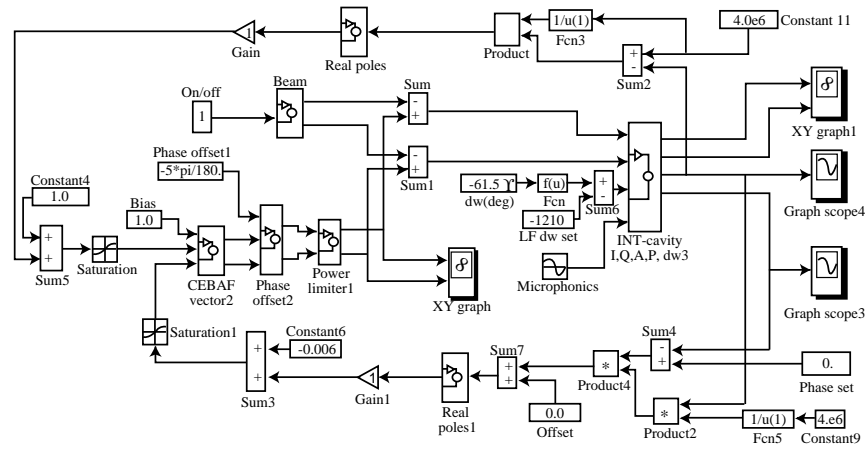

Figure. 1. RF control system model
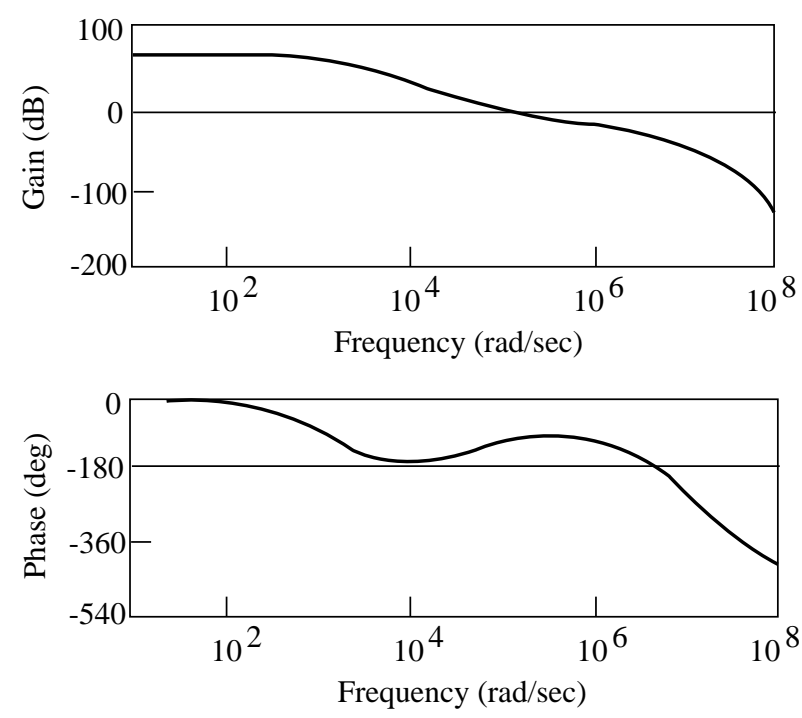

Figure. 2. Bode plot of CEBAF's rf control system

recover from the induced perturbance and establish steady-state in less than $0.1 \mathrm{msec}$ (to the $10^{-2}$ level of error suppression).

\section{c) Control of microphonics and current fluctuations}

We observed a reduction of microphonics by a factor of 30 after the low-frequency gain was turned on. At steady-state the phase of the accelerating field fluctuates at the $0.02^{0}$ level peak-to-peak and the amplitude at the $1 \times 10^{-5}$ level; therefore the system exceeds performance requirements. Finally we modulated the beam current by $2 \%$ peak-to-peak at a frequency of $1 \mathrm{kHz}$. The response of the system is still dominated by microphonic noise, therefore $2 \% \mathrm{p}-\mathrm{p}$ current fluctuations at $1 \mathrm{kHz}$ are entirely within the range of control of the system.

\section{References}

[1] D. Neuffer et al., these proceedings (1995)

[2] S. Simrock, proceedings PAC 1991

[3] SIMULINK, The Mathworks, Inc.

[4] P. B. Wilson, SLAC-PUB-2884, February 1982

[5] A. Mosnier, J. Tessier, TESLA 94-16, May 1994

[6] G. Krafft, S. Simrock, K. Mahoney, proc. PAC 1991

[7] L. Merminga et al., proceedings PAC 1993 